\title{
Conductividad eléctrica y difusión de oxígeno en el sistema Bifevox
}

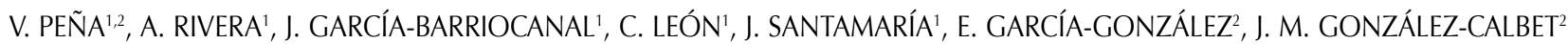 \\ ' GFMC, Dpto. Física Aplicada III, Facultad de Físicas, Universidad Complutense de Madrid. \\ ${ }^{2}$ Dpto. Química Inorgánica, Facultad de Químicas, Universidad Complutense de Madrid.
}

\begin{abstract}
Presentamos medidas de la conductividad eléctrica del sistema BIFEVOX $\quad \mathrm{Bi}_{4} \mathrm{~V}_{2-\mathrm{x}} \mathrm{Fe}_{\mathrm{x}} \mathrm{O}_{11-\mathrm{y}}(0 \leq \mathrm{x} \leq 0.9 ; 0 \leq \mathrm{y} \leq 1)$, en el que se realiza la sustitución de iones V (IV) por Fe (III) de forma sistemática. La conductividad muestra un comportamiento potencial con la frecuencia, descrito por $\sigma^{*}(\omega)=\sigma_{\mathrm{dc}}\left[1+\left(\mathrm{j} \omega / \omega_{\mathrm{p}}\right)^{\mathrm{n}}\right]$, y conocido como respuesta dieléctrica universal. Análogamente, el módulo eléctrico presenta picos asimétricos, cuya función de relajación en el dominio del tiempo puede describirse mediante exponenciales "estiradas" de la forma $\phi(t)=\exp \left(-\left(t / \tau_{\sigma}\right)^{\beta}\right) . \beta$ da cuenta del grado de correlación del transporte iónico, siendo su valor, $\beta=0.56 \pm 0.03$, casi independiente de la temperatura y del contenido en Fe. Con el aumento en el contenido de $\mathrm{Fe}$, la conductividad disminuye exponencialmente y la energía de activación del proceso de conducción aumenta de 0.20 a 0.97 eV. Estos resultados se discuten en términos de la ordenación de vacantes oxígeno al dopar con Fe (III).
\end{abstract}

Palabras clave: conductor iónico, difusión de oxigeno, espectroscopía de impedancias, correlación iónica.

\section{Electrical conductivity and oxygen diffusion in Bifevox.}

We present electrical conductivity measurements of BIFEVOX $\mathrm{Bi}_{4} \mathrm{~V}_{2-\mathrm{x}} \mathrm{Fe}_{\mathrm{x}} \mathrm{O}_{11-\mathrm{y}}(0 \leq \mathrm{x} \leq 0.9 ; 0 \leq \mathrm{y} \leq 1)$, in which $\mathrm{V}$ (IV) ions have been systematically substituted by $\mathrm{Fe}(\mathrm{III})$ ions. Conductivity shows a power law frequency dependence described by the form $\sigma^{*}(\omega)=\sigma_{\mathrm{dc}}\left[1+\left(\mathrm{j} \omega / \omega_{\mathrm{p}}\right)^{\mathrm{n}}\right]$, known as universal dynamic response. Conversely, the electric modulus shows asymmetric peaks, characterized by stretched exponentials relaxation functions in time domain of the form $\phi(t)=\exp \left(-\left(t / \tau_{\sigma}\right)^{\beta}\right)$. $\beta$ is determined by the degree of correlation in the ionic motion. It's value, $\beta=0.56 \pm 0.03$, is almost independent of temperature and iron content. Increasing Fe content leads to an exponential decrease of the conductivity and to an increase of the activation energy of the conduction process from 0.20 to $0.97 \mathrm{eV}$. These results are discussed in terms of oxygen vacancy ordering upon Fe (III) substitution.

Keywords: ionic conductor, oxygen diffusion, impedance spectroscopy, ionic correlation

\section{INTRODUCCIÓN}

Durante los últimos años se ha producido un creciente interés en el estudio de los sólidos conductores iónicos, motivado por la utilidad de estos materiales en la fabricación de dispositivos, como pilas de combustible, sensores, o baterías de estado sólido. Para mejorar las prestaciones de estos dispositivos es necesario conocer los mecanismos de transporte de los materiales que los forman, así como buscar nuevos materiales que mejoren las características de los que actualmente se conocen. Entre todos los conductores iónicos, los que presentan un futuro más prometedor son quizás los conductores de hidrógeno y de oxígeno, por su aplicación a las células de combustible. En concreto, los conductores de oxígeno ya se emplean industrialmente en prototipos de centrales eléctricas basadas en pilas de combustible de alta temperatura, pero la deseada posibilidad de disminuir la temperatura de funcionamiento se ve limitada por la baja conductividad de oxígeno a temperatura ambiente de los materiales usados como electrolitos.

El óxido $\mathrm{Bi}_{4} \mathrm{~V}_{2} \mathrm{O}_{11}$, conductor iónico por vacantes oxígeno, es el primer miembro de la familia de óxidos de Aurivillius $(\mathrm{m}=1)$, con fórmula general $\left(\mathrm{Bi}_{2} \mathrm{O}_{2}\right)\left(\mathrm{A}_{\mathrm{m}-1} \mathrm{~B}_{\mathrm{m}} \mathrm{O}_{3 \mathrm{~m}+1}\right)$. Esta fase posee vacantes oxígeno (1), y tiene una alta conductividad iónica en su fase tetragonal $\gamma(0.3$ $\mathrm{S} \mathrm{cm}^{-1}$ a $1000 \mathrm{~K}$ ) debido a la alta movilidad de los iones oxígeno. La estructura de este compuesto se puede describir como constituido por capas de $\left(\mathrm{Bi}_{2} \mathrm{O}_{2}\right)^{2+}$ aislantes, alternadas con capas tipo perovskita, de composición $\left(\mathrm{VO}_{3.5}\right)^{2-}$, siendo las capas $\mathrm{V}-\mathrm{O}$ deficientes en oxígeno y responsables de la conductividad iónica. Además el $\mathrm{Bi}_{4} \mathrm{~V}_{2} \mathrm{O}_{11}$ muestra transiciones de fase reversibles $\alpha \leftrightarrow \beta$ y $\beta \leftrightarrow \gamma$ que ocurren a 680 y 840
$\mathrm{K}$ respectivamente, cada una de las cuales está asociada a un aumento del desorden de las vacantes oxígeno. Por encima de $840 \mathrm{~K}$ dicho desorden hace que el material se comporte como un buen conductor aniónico (2). Resultó por tanto muy interesante la estabilización de la fase $\gamma$ a temperatura ambiente mediante la sustitución del vanadio por otros iones metálicos, lo que dio lugar a una nueva familia de materiales denominados BIMEVOX $(3,4)$. Los estudios de la conductividad eléctrica de las series BIMEVOX se han centrado en la determinación de los valores de la conductividad dc frente a la temperatura, quedando sin resolver las cuestiones relacionadas con la dinámica del proceso de conducción. La mayor parte de los procesos de dopaje investigados generan compuestos con estequiometría aniónica por debajo de $\mathrm{O}_{11}$ creando vacantes de oxígeno y favoreciendo la movilidad de éste en los planos V-O $(3,4)$. Sin embargo, modificaciones estructurales en el proceso de sustitución, y la capacidad del catión dopante para afectar el orden a largo alcance del compuesto de origen pueden afectar al previsible aumento de la conductividad iónica al insertar metales en $\mathrm{Bi}_{4} \mathrm{~V}_{2} \mathrm{O}_{11}(5)$. El estudio de la correlación de las vacantes de oxígeno en el proceso de conducción de la serie BIFEVOX es de gran interés, por tratarse de un material de dimensionalidad reducida por su estructura laminar, y que sufre ordenaciones de vacantes al insertar Fe (III) (6).

La técnica experimental más usada en el estudio de la correlación entre iones en los procesos de conducción iónica es la espectroscopía de impedancias. Es bien conocido que el movimiento iónico correlacionado da lugar a una dependencia potencial característica de la conductividad ac, conocida como "respuesta dieléctrica universal" (7), y 
descrita por la expresión de Jonscher:

$$
\sigma^{*}(\omega)=\sigma_{\mathrm{dc}}\left[1+\left(j \omega / \omega_{\mathrm{p}}\right)^{\mathrm{n}}\right]
$$

donde $\sigma_{d c}$ es la conductividad dc y $\omega_{p}$ es una frecuencia de cruce, ambas activadas térmicamente con la misma energía de activación. La dependencia potencial de la conductividad con la frecuencia aparece como resultado de un mecanismo de conducción por saltos no aleatorios, y $n$ es un exponente fraccionario que da cuenta del grado de correlación de los iones, siendo igual a cero para saltos aleatorios y no correlacionados. La respuesta en el tiempo se obtiene a partir del módulo eléctrico $M^{*}(\omega)=1 / \varepsilon^{*}(\omega)=j \omega \varepsilon_{0} / \sigma^{*}(\omega)$, magnitud que se puede expresar en términos de una función de relajación $\phi(t)$, de acuerdo con la expresión (8):

$$
M^{*}(\omega)=\frac{1}{\varepsilon_{\infty}}\left[1-\int_{0}^{\infty}\left(-\frac{d \phi}{d t}\right) e^{-j \omega t} d t\right]
$$

donde $\varepsilon_{\infty}$ es el valor de la permitividad dieléctrica para altas frecuencias. Es habitual describir la función de relajación como una exponencial "estirada", conocida como función de Kohlrausch-Williams-Watts (KWW) (9):

$$
\phi(t)=\exp \left(-\left(t / \tau_{\sigma}\right)^{\beta}\right)
$$

El tiempo de relajación característico $\tau_{\sigma}$ es aproximadamente el inverso de la frecuencia de cruce $\omega_{p}$ en la eq. $1, \tau_{\sigma} \approx 1 / \omega_{p^{\prime}}$ y por tanto está activado térmicamente con la misma energía de activación que la conductividad dc. El exponente $\beta$ está relacionado con el grado de correlación de los iones en su movimiento, y por tanto con $n$, de forma que $\beta \approx 1-n$ (10). La representación del módulo eléctrico es ampliamente utilizada para el análisis del movimiento correlacionado de los iones ya que es menos sensible a los fenómenos de bloqueo (11-13). Es más, la función de relajación obtenida a partir de la representación del módulo eléctrico de los datos experimentales está directamente relacionada con la dinámica microscópica de la conducción por saltos de iones (14).

En este artículo presentamos medidas de la conductividad eléctrica en el sistema BIFEVOX $\mathrm{Bi}_{4} \mathrm{~V}_{2-\mathrm{x}} \mathrm{Fe}_{\mathrm{x}} \mathrm{O}_{11-\mathrm{y}}(0 \leq \mathrm{x} \leq 0.9 ; 0 \leq \mathrm{y} \leq 1)$, en el que los iones de $\mathrm{V}$ (IV) se han sustituido progresivamente por iones Fe (III) hasta un contenido de 0.9 por fórmula. Los resultados obtenidos en la conductividad iónica se discuten en términos de los estudios microestructurales efectuados (6), que indican la aparición de dos tipos de dominios estructurales distintos que difieren en la ordenación de las vacantes oxígeno de las capas $\mathrm{V}(\mathrm{Fe})-\mathrm{O}$.

\section{DESCRIPCIÓN EXPERIMENTAL}

Las muestras de composición $\mathrm{Bi}_{4} \mathrm{~V}_{2-\mathrm{x}} \mathrm{Fe}_{\mathrm{x}} \mathrm{O}_{11-\mathrm{y}}(0 \leq \mathrm{x} \leq 0.9,0 \leq \mathrm{y} \leq 1)$, se prepararon por reacción en estado sólido a partir de las cantidades estequiométricas de $\mathrm{Bi}_{2} \mathrm{O}_{3}$ (Merck, 99.9\%), $\mathrm{V}_{2} \mathrm{O}_{5}$ (Merck 99.9\%) y $\mathrm{Fe}_{2} \mathrm{O}_{3}$ (Merck 99.9\%). Los detalles del procedimiento de síntesis, y la caracterización química y microestructural ya han sido publicados en la referencia 6 .

Las medidas de espectroscopía de impedancias se realizaron utilizando los analizadores de impedancias, HP4284A y HP4285A, controlados automáticamente con un PC mediante un bus GPIB-IEEE. El rango de frecuencias empleado fue $20 \mathrm{~Hz}$ - $30 \mathrm{MHz}$, y el de temperaturas $213-497 \mathrm{~K}$. Las muestras policristalinas de BIFEVOX se prensaron en forma de pastillas cilíndricas con un diámetro de $8 \mathrm{~mm}$ y espesor variable entre 1 y $1.5 \mathrm{~mm}$, y se depositaron electrodos de plata. Todas las medidas eléctricas fueron realizadas bajo un flujo permanente de gas nitrógeno para garantizar una atmósfera inerte.

\section{RESULTADOS Y DISCUSIÓN}

La figura 1 muestra la dependencia de la parte real de la conductividad de una muestra representativa de toda la serie, $\mathrm{Bi}_{4} \mathrm{~V}_{1.8} \mathrm{Fe}_{0.2} \mathrm{O}_{10.8^{\prime}}$ para temperaturas entre 213 y $497 \mathrm{~K}$. A altas temperaturas y bajas frecuencias se observa una zona de conductividad constante o plateau que corresponde a la conductividad dc o bulk del material. A mayores frecuencias tenemos una dependencia potencial de la conductividad con la frecuencia, siendo $\omega_{p}$ la frecuencia de cruce. La líneas en la figura son el ajuste a la eq. (1), que permite obtener los valores de $\sigma_{d c}$ y $\omega_{p}$ en función de la temperatura. Con la disminución de la temperatura, la frecuencia de cruce de la dependencia potencial se desplaza hacía frecuencias menores, y la conductividad dc disminuye, estando ambos parámetros activados con la misma energía de activación $E_{\sigma}$. A menores temperaturas, el plateau dc sale de nuestra ventana experimental por las bajas frecuencias, y el término potencial de la conductividad ac cambia a una contribución de dependencia casi lineal con la frecuencia, debido a la aparición de otro régimen diferente. Esta contribución a la conductividad consiste en unas pérdidas dieléctricas casi independientes de la frecuencia $\varepsilon^{\prime \prime} \approx A$, que domina la conductividad ac en conductores iónicos a suficientes bajas temperaturas o altas frecuencias (15-20). La figura 2 muestra la dependencia de la parte imaginaria

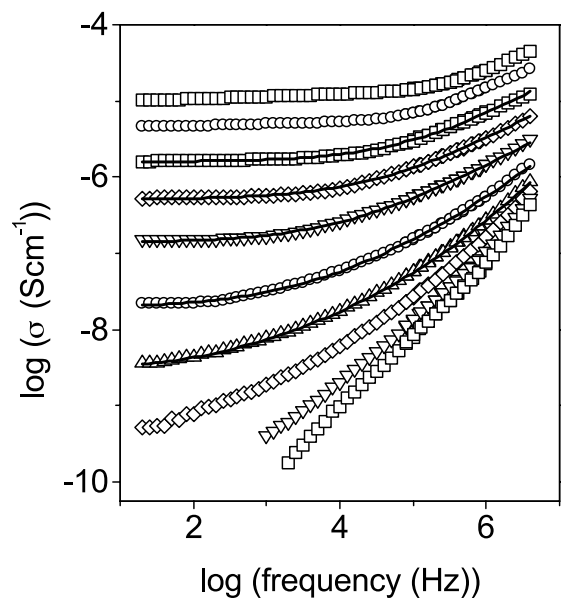

Figura 1. Parte real de la conductividad frente a la frecuencia del $\mathrm{Bi}_{4} \mathrm{~V}_{1.8} \mathrm{Fe}_{0.2} \mathrm{O}_{10.8}$ para varias temperaturas (de arriba hacia abajo en la figura: $497,467,437,407,377,338,308,278,243$ y $213 \mathrm{~K}$ ).

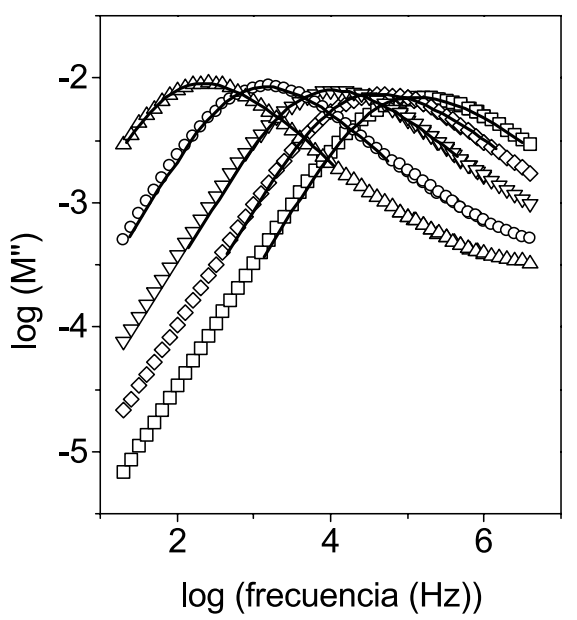

Figura 2. Parte imaginaria del módulo eléctrico frente a la frecuencia del $\mathrm{Bi}_{4} \mathrm{~V}_{1.8} \mathrm{Fe}_{0.2} \mathrm{O}_{10.8}$ en escala doble logarítmica para varias temperaturas $(437 \mathrm{~K}(\square)$, $407 \mathrm{~K}(\diamond), 377 \mathrm{~K}(\nabla), 338 \mathrm{~K}(\mathrm{\nabla})$ y $308 \mathrm{~K}(\Delta))$. Las líneas son el ajuste a las expresiones (2) y (3) del texto, mostrando las funciones de relajación KWW en el dominio del tiempo. 
del módulo eléctrico $M^{\prime \prime}(\omega)$, para varias temperaturas. Las líneas son el ajuste a las ecuaciones (2) y (3). En el rango de temperatura en el que se observa completo el máximo del módulo (308 - 437 K), se han obtenido valores de $\beta$ cercanos a 0.56 , con pequeñas variaciones con la temperatura entre 0.54 y 0.58 . Para las muestras con distinto contenido en Fe (III) se han obtenido también valores muy similares de $\beta=0.56 \pm 0.03$. En esta representación del módulo eléctrico, el régimen de "pérdidas constantes" aparece en la rama de alta frecuencia del máximo, que tiende hacia un plateau a las frecuencias más altas y temperaturas más bajas. La frecuencia del máximo está activada con la misma energía de activación que la conductividad dc obtenida de la figura 1 , y la intensidad del mismo, que viene determinada por $\beta$ y por el parámetro $\varepsilon_{\alpha}$, presenta valores similares en todas las muestras, con $\varepsilon_{\infty}=30 \pm 5$ casi independiente del contenido de Fe (III).

La figura 3 muestra la dependencia de la conductividad dc con la temperatura en un gráfico tipo Arrhenius, para varios contenidos de $\mathrm{Fe}$ (III) en la serie $\mathrm{Bi}_{4} \mathrm{~V}_{2-\mathrm{x}} \mathrm{Fe}_{\mathrm{x}} \mathrm{O}_{11-\mathrm{y}}(0 \leq \mathrm{x} \leq 0.9,0 \leq \mathrm{y} \leq 1)$. Se observa que la energía de activación del proceso de conducción iónica aumenta con el contenido de Fe (III) de 0.20 a 0.97 eV. El aumento de la energía de activación da lugar a una disminución exponencial de la conductividad dc a una temperatura dada, al aumentar el contenido en hierro, como muestra la figura 4 . Es más, hemos encontrado que la energía de activación de la conductividad dc aumenta linealmente con el contenido de Fe (III) (ver figura 5). Este comportamiento no se puede explicar en términos de un cambio en la concentración de portadores, que justificaría una variación lineal de la conductividad con el contenido en hierro, sino que requiere una explicación a partir de los cambios microestructurales que están teniendo lugar al dopar con $\mathrm{Fe}$ (III). La sustitución de iones $\mathrm{V}$ (IV) por iones Fe (III) en el $\mathrm{Bi}_{4} \mathrm{~V}_{2} \mathrm{O}_{11}$ introduce vacantes oxígeno, que se acomodan en dominios con diferente estequiometría en oxígeno. Además de la matriz $\mathrm{Bi}_{4} \mathrm{~V}_{2} \mathrm{O}_{11}$ con vacantes oxígeno desordenadas, aparecen dos dominios que presentan estequiometría $\mathrm{O}_{10.66} \mathrm{y} \mathrm{O}_{10^{\prime}}$ llamados $\mathrm{y}$ y respectivamente en la figura 6. Ambos tipos de dominios aumentan su tamaño al incrementar el contenido de Fe (III), a expensas de la matriz altamente conductora. El dominio con estequiometría aniónica $\mathrm{O}_{10.66}$ se caracteriza por una

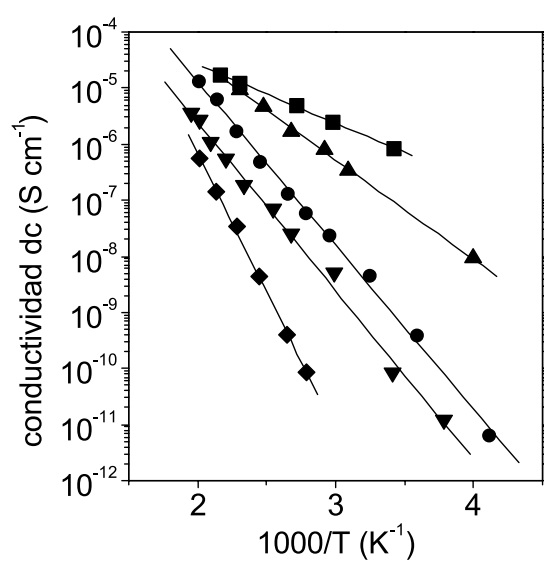

Figura 3. Gráfico Arrhenius de la conductividad dc para distintos contenidos de $\mathrm{Fe}$ (III) en la serie BIFEVOX $\mathrm{Bi}_{4} \mathrm{~V}_{2-x} \mathrm{Fe}_{\mathrm{x}} \mathrm{O}_{11-v^{*}}$ Las energías de activación son $\mathrm{E}_{\sigma}=0.20 \mathrm{eV}$ para $\mathrm{x}=0(\mathrm{\square}), \mathrm{E}_{\mathrm{\sigma}}=0.35 \mathrm{eV}$ para $\mathrm{x}=0.1(\mathbf{\Delta})$, $\mathrm{E}_{\sigma}=0.56 \mathrm{eV}$ para $\mathrm{x}=0.2(\mathrm{Q}), \mathrm{E}_{\sigma}=0.60 \mathrm{eV}$ para $\mathrm{x}=0.5(\boldsymbol{\nabla}), \mathrm{y} \mathrm{E}_{\sigma}=0.97 \mathrm{eV}$ para $\left.\mathrm{x}=0.9(\mathrm{C})\right)$.

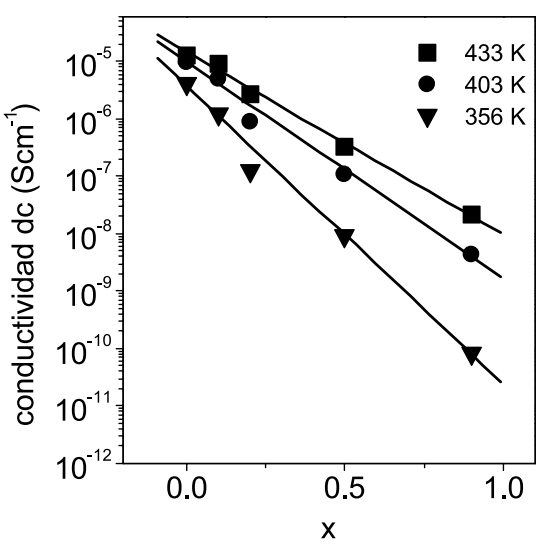

Figura 4. Conductividad dc frente a contenido de Fe para temperaturas fijas: 356 $\mathrm{K}(\boldsymbol{\nabla}), 403 \mathrm{~K}(\mathrm{\square})$ y $433 \mathrm{~K}(\mathrm{\square})$. A destacar el descenso exponencial de la conductividad dc frente al contenido de Fe (III).

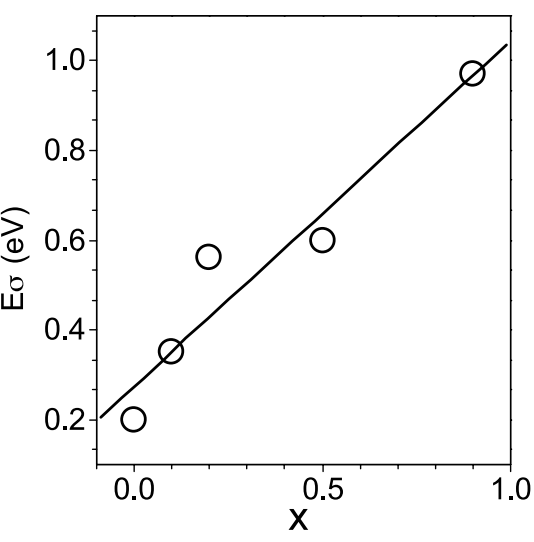

Figura 5. Energía de activación de la conductividad dc frente al contenido de Fe (III), la línea es el ajuste lineal.

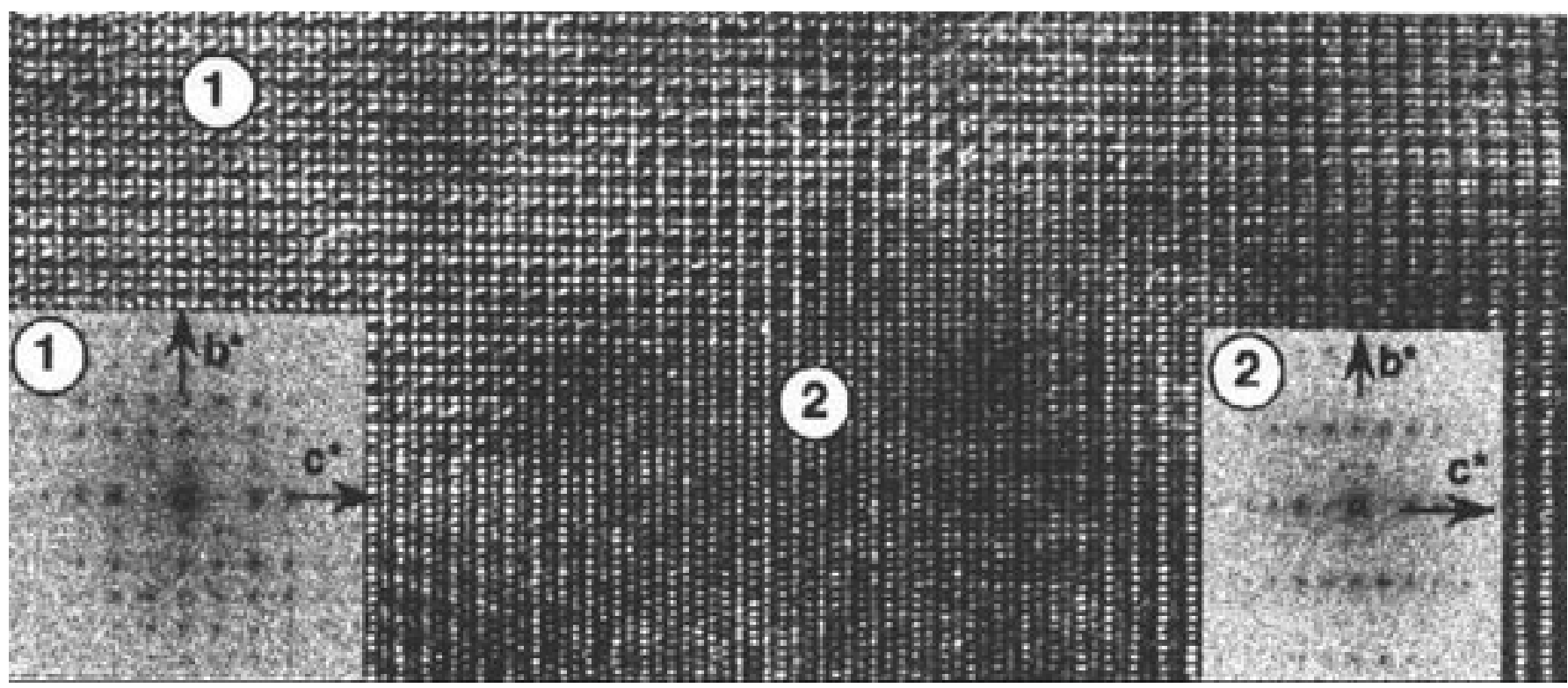

Figura 6. Imagen de microscopía electrónica de alta resolución para la muestra de composición $x=0.7$, tomada en el eje de zona [100]. Los dominios $\mathrm{\square}$ y $\mathrm{se}$ corresponden con los diagramas de difracción que se incluyen en la figura. 
ordenación de las vacantes, dando lugar a una superestructura de orden tres a lo largo del eje b. Esta ordenación de vacantes daría lugar a una secuencia ordenada de dos pirámides y un octaedro a lo largo de [010], lo que supone un importante impedimento en la difusión del oxígeno. Esto queda reflejado en un aumento de la energía de activación. En el dominio de composición $\mathrm{O}_{10^{\prime}}$ la capa perovskita puede considerarse constituida por pirámides de base cuadrada (21) como en el compuesto $\mathrm{Bi}_{4} \mathrm{~V}_{2} \mathrm{O}_{10^{\prime}} \mathrm{y}$ las posiciones de oxígeno que antes eran vacantes, se convierten en posiciones vacías. Para el salto de los iones, está situación es equivalente a tener todos los átomos metálicos en coordinación octaédrica, es decir, sin vacantes aniónicas, como en el compuesto $\mathrm{Bi}_{4} \mathrm{MoO}_{12}$ (22). Por ello, se espera que la conductividad esté fuertemente restringida en estos dominios, disminuyendo su contribución al mecanismo de conducción.

La existencia de dominios con una conductividad diferente sugiere un complicado escenario en el que las vacantes oxígeno difunden a través de los dominios más conductores formando un sistema controlado por percolación. El incremento en tamaño del dominio $\mathrm{O}_{10^{\prime}}$ con una pequeña contribución a la conductividad, y del dominio $\mathrm{O}_{10.66^{\prime}}$ con una movilidad reducida del oxígeno, da lugar a un aumento de la energía de activación de la conductividad dc. Para la muestra con un contenido $\mathrm{x}=0.9$ el material tiene una estequiometría aniónica muy cercana a $\mathrm{O}_{10^{\prime}}$ y las vacantes oxígeno se convierten en posiciones inaccesibles para los átomos oxígeno (21). Como resultado, esta muestra presenta una alta energía de activación de $0.97 \mathrm{eV}$ y un valor extremadamente bajo de la conductividad a temperatura ambiente, menor de $10^{-12} \mathrm{Scm}^{-1}$.

\section{CONCLUSIONES}

En resumen, hemos presentado un estudio de la conductividad iónica por vacantes de oxígeno de la serie BIFEVOX mediante espectroscopía de impedancias. Se ha realizado un análisis de la conductividad ac y del módulo eléctrico, mostrando dependencias potenciales en el dominio de la frecuencia. Hemos encontrado que la función de correlación de los portadores de carga se puede describir mediante exponenciales estiradas de la forma $\phi(t)=\exp \left(-\left(t / \tau_{\sigma}\right)^{\beta}\right)$. La relajación de la conductividad eléctrica en el dominio del tiempo presenta un exponente $\beta$ distinto de 1 como consecuencia de los efectos de correlación en la difusión del ion oxígeno, y que permanece casi constante, independientemente de la temperatura y contenido en Fe (III). Además, se ha encontrado que a temperatura ambiente la conductividad dc disminuye de forma exponencial más de 5 órdenes de magnitud al aumentar el contenido de Fe (III), a la vez que la energía de activación de la conductividad aumenta. Estos resultados se han discutido en términos del ordenamiento de las vacantes oxígeno debido a la aparición de dos dominios con diferente estequiometría aniónica, al sustituir los iones V (IV) por iones Fe (III). En estos nuevos dominios, cuyo tamaño relativo aumenta con el contenido de Fe (III), la movilidad del oxígeno está más restringida debido a consideraciones microestructurales.

\section{BIBLIOGRAFÍA}

1. A. A. Bush, V. G. Koshelayeva, Yu. N. Venevtsev, "Crystals of the $\mathrm{Bi}_{2} \mathrm{GeO}_{5}$ - $\mathrm{Bi}_{4} \mathrm{~V}_{2} \mathrm{O}_{11}$ system". J. Appl. Phys. 1985, 24, 625.

2. F. Abraham, M. F. Debreuille, G. Mairesse, G. Nowogrocki, "Phase transitions and ionic conductivity in $\mathrm{Bi}_{4} \mathrm{~V}_{2} \mathrm{O}_{11}$. An oxide with a layered strucuture". Solid State Ionics 1988, 28-30, 529.

3. T. Iharada, A. Hammouche, J. Fouletier, M. Kleitz, J. C. Boivin, G. Mairesse, "Electrochemical characterization of BIMEVOX oxide-ion conductors". Solid State Ionics 1991, 48, 257.

4. F. Abraham, J. C. Boivin, G. Mairesse, G. Nowogrocki, "The bimevox series: A new family of high performance oxide ion conductors".Solid State Ionics 1990, 40-41, 934.

5. V. Peña, A. Rivera, C. León, J. Santamaría, E. García-González, v GonzálezCalbet, "Correlated oxygen difusión in BIFEVOX". Chem. Mater. 2002, 14, 1606.

6. E. García-Gonzalez, M. Arribas, J. M. González-Calbet. “Short-range longrange order transfomation in the $\mathrm{Bi}_{4} \mathrm{~V}_{2-\mathrm{x}} \mathrm{Fe}_{\mathrm{x}} \mathrm{O}_{11-\mathrm{y}}$ series". Chem. Mater 2001, 13, 96.

7. A. K. Jonscher, en Dielectric Relaxation in Solids (Chelsea Dielectric, London, 1983).

8. P. B. Macedo, C. T. Moyniham, R. Bose. "The role of ionic diffusion in polarization in vitreous ionic conductors". Phys Chem Glasses. 1972 13, 171.

9. R. Kohlrausch, "Nachtrag über die elastiche Nachwirkung beim Cocon und Glasladen". Ann. Phys. (Leipzig) 1847, 72, 393.

10. K. L. Ngai, "Universality of low-frequency fluctuation, dissipation and relaxation properties of condensed matter. I". Comments Solid State Phys. 1979, 9, 121.

11. C. León, M. L. Lucia, J. Santamaría, M. A. Paris, J. Sanz, A. Várez, “Electrical conductivity relaxation and nuclear magnetic resonance of Li conducting $\mathrm{Li}_{0.5} \mathrm{La}_{0.5} \mathrm{TiO}_{3}^{\prime \prime}$.Phys. Rev. B., 1996, 54, 184 .

12. C. León, J. Santamaría, M. A. Paris, J. Sanz, J. Ibarra, L. M. Torres, “NonArrhenius conductivity in the fast ionic conductor $\mathrm{Li}_{0.5} \mathrm{La}_{0.5} \mathrm{TiO}_{3}$ : Reconciling spin-lattice and electrical-conductivity relaxations".Phys. Rev. B , 1997, 56, 5302.

13. A. Rivera, J. Santamaria, C. León, “Electrical conductivity relaxation in thinfilm yttria-stabilized zirconia". Appl. Phys. Lett., 2001, 78, 610.

14. K.L. Ngai, G.N. Greaves, C.T. Moynihan, “Correlation between the activation energies for ionic conductivity for short and long time scales and the Kohlrausch stretching parameter beta for ionically conducting solids ans melts".Phys.Rev.Lett., 1998, 80, 1018.

15. K. L. Ngai, C. León, "Relating macroscopic electrical relaxation to microscopic movements of the ions in ionically conducting materials by theory and experiment". Phys. Rev. B, 1999, 60, 9396.

16. W. K. Lee, J. F. Liu, A. S Nowick, "Limiting behavior of ac conductivity in ionically conducting crystals and glasses: A new universality".Phys. Rev. Lett. 1991, 67, 1559.

17. C. León, A. Rivera, A. Várez, J. Sanz, J. Santamaría, K. L. Ngai, “Origin of constant loss in ionic conductors".Phys. Rev. Lett. 2001, 86, 1279.

18. A. Rivera, C. León, C. P. E. Varsamis, G. D. Chryssikos, K. L. Ngai, C. M. Roland, J. L. Buckley, "Cation mass dependence of the nearly constant dielectric loss in alkali triborate glasses".Phys. Rev. Lett., 2002, 88, 125902.

19. C. León, A. Rivera, J. Santamaría, C. T. Moynihan, K. L. Ngai, Comment on "Ionic conduction in glass: new information on the interrelation between the Jonscher behavior and the Nearly Constant-Loss behavior from broadband conductivity spectra". Phys. Rev. Lett., 2002, 89, 079601.

20. C. León, P. Lunkenheimer, K. L. Ngai, “Test of universal scaling of ac conductivity in ionic conductors". Phys. Rev. B, 2001, 64, 184304.

21. E. García-Gonzalez, M. Arribas, J. M. González-Calbet, “Oxygen content and microstructure in g- $\mathrm{Bi}_{4} \mathrm{~V}_{2} \mathrm{O}_{11}$ ".J. Mat. Chem. 2001, 11, 2320.

22. K. S. Knight, "The crystal structure of russellite; a re-determination using neutron powder diffraction of synthetic $\mathrm{Bi}_{2} \mathrm{WO}_{6}{ }^{\prime \prime}$. Mineral. Mag. 1992, 56, 339 .

Recibido: 01.02 .03

Aceptado: 30.11 .03 